\title{
The Knotted Sky II: Does BICEP2 require a nontrivial primordial power spectrum?
}

\author{
Kevork N. Abazajian, ${ }^{a}$ Grigor Aslanyan, ${ }^{b}$ Richard Easther, ${ }^{b}$ and \\ Layne C. Price ${ }^{b}$
}

${ }^{a}$ Department of Physics, University of California at Irvine, Irvine, CA 92697

${ }^{b}$ Department of Physics, University of Auckland, Private Bag 92019, Auckland, New Zealand E-mail: kevork@uci.edu,g.aslanyan@auckland.ac.nz,r.easther@auckland.ac.nz, lpri691@aucklanduni.ac.nz

\begin{abstract}
An inflationary gravitational wave background consistent with BICEP2 is difficult to reconcile with a simple power-law spectrum of primordial scalar perturbations. Tensor modes contribute to the temperature anisotropies at multipoles with $l \lesssim 100$, and this effect — together with a prior on the form of the scalar perturbations - was the source of previous bounds on the tensor-to-scalar ratio. We compute Bayesian evidence for combined fits to BICEP2 and Planck for three nontrivial primordial spectra: a) a running spectral index, b) a cutoff at fixed wavenumber, and c) a spectrum described by a linear spline with a single internal knot. We find no evidence for a cutoff, weak evidence for a running index, and significant evidence for a "broken" spectrum. Taken at face-value, the BICEP2 results require two new inflationary parameters in order to describe both the broken scale invariance in the perturbation spectrum and the observed tensor-to-scalar ratio. Alternatively, this tension may be resolved by additional data and more detailed analyses.
\end{abstract}




\section{Contents}

1 Introduction 1

2 Method 3

2.1 Likelihoods, priors, and Bayesian evidence 3

2.2 Non-power-law scalar spectrum 3

3 Results $\quad 8$

4 Discussion $\quad 9$

\section{Introduction}

The BICEP2 experiment $[1,2]$ has reported a detection of primordial B-modes in the cosmic microwave background (CMB). ${ }^{1}$ The most natural explanation for a B-mode signal is a stochastic background of long-wavelength gravitational waves, or tensor perturbations [4]. This constitutes strong prima facie evidence for an inflationary phase in the early universe, the most widely-studied source for a stochastic background of gravitational waves. If the observational data and theoretical explanation are confirmed, the B-mode signal will provide unprecedented insight into the mechanism responsible for inflation.

The measured tensor-to-scalar ratio has a $68 \%$ confidence-interval $(\mathrm{CI})$ of $r=0.20_{-0.05}^{+0.07}$ and differs from zero with a statistical significance of $5.9 \sigma$. However, the temperature data from Planck [5-7], SPT [8], and ACT [9], combined with WMAP [10] polarization, yields $r \lesssim 0.11$ at the $95 \% \mathrm{CI}$, in significant tension with the BICEP2 result.

There are several potential explanations for this discrepancy. The first is that the BICEP2 analysis over-estimates the amplitude of the B-mode itself [11]. The second possibility is that the primordial B-mode is accurately measured, but sourced by a mechanism unrelated to the standard assumptions for the primordial inflationary phase [12-21]. Conversely, existing CMB data may have been misanalysed, although this appears unlikely given the agreement of Planck with WMAP at large and intermediate scales and with ACT and SPT at small scales.

Another suggestion is that the large-scale scalar power spectrum is suppressed relative to that predicted by the best-fit $\Lambda$ CDM scenario. Pre-BICEP2 constraints on the inflationary gravitational wave background were driven primarily by the contribution of tensor modes to the temperature-temperature (TT) anisotropies. This is illustrated in Fig. 1, which shows the contribution to $C_{l}^{T T}$ at low- $l$ from a tensor background with $r=0.2$. The tensor contribution to individual $C_{l}^{T T}$ is small, but systematically increases the TT multipoles for all $l \lesssim 100$. However, to constrain the primordial tensor background using this signal we must have an independent estimate of the contributions $C_{l}^{T T}$ from scalar perturbations alone. Moreover, the measured low-multipole $C_{l}^{T T}$ typically lie below the best fit values for simple power-law spectra, so the inclusion of a tensor background is likely to reduce the likelihood relative to $r=0$. Fig. 1 also shows a sample angular power spectrum derived from a primordial scalar spectrum with a sharp cut-off in power at a comoving wavenumber $k=0.002 \mathrm{Mpc}^{-1}$. This

\footnotetext{
${ }^{1}$ B-mode polarization from CMB lensing was detected earlier by the POLARBEAR experiment [3].
} 

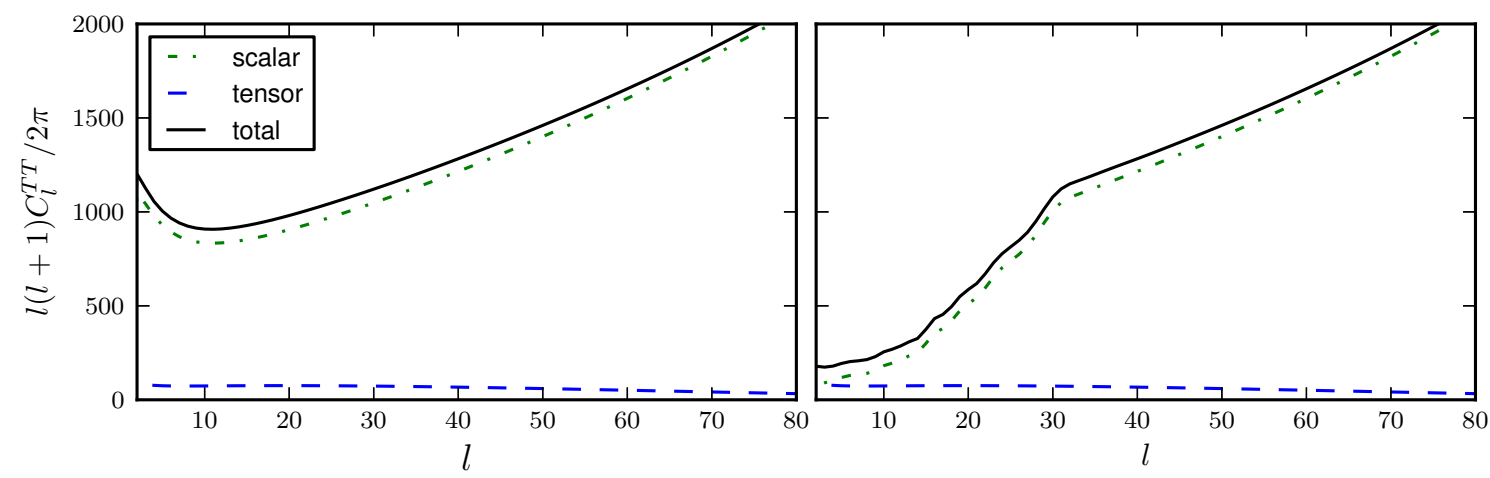

Figure 1. The contribution to the (lensed) TT power spectrum from scalar and tensor modes $(r=0.2)$. The left panel shows a standard power law primordial power spectrum with typical values of the cosmological parameters. The right panel shows the same primordial power spectrum but cut-off below $k=0.002 \mathrm{Mpc}^{-1}$ (corresponding to $l \sim 30$ ).

particular scenario has a very low likelihood relative to the Planck and WMAP datasets, but provides an extreme illustration of how a scalar spectrum with a cutoff could compensate for a tensor contribution to the $C_{l}^{T T}$ for $l \lesssim 100$.

In this paper we focus on the implications of the BICEP2 result for the scalar power spectrum $\Delta^{2}(k)$, performing joint analyses of the BICEP2 and Planck datasets. We consider three possibilities: (i) a running spectral index, (ii) a sharp cut-off in power at scale $k_{\text {cut }}$, and (iii) a discontinuity in the spectral index at scale $k_{\text {knot }}$. The latter two scenarios are implemented via the algorithm described by us in Ref. [22].

The BICEP2 analysis [1,2] presents joint constraints from BICEP2 and Planck with a running index, but focusses primarily on the polarization and B-mode amplitude and does not discuss the issue in detail. We reproduce the BICEP2 constraints on a running index, and compute Bayesian evidence (relative to $\Lambda \mathrm{CDM}$ ) of $\Delta \log Z=1.1$ for the running case. The cutoff spectrum does not give a significant improvement, since it suppresses the scalar power by a factor far larger than the corresponding increase in power due to tensor contributions. Finally, a break in the spectral index - implemented as a splined $P(k)$ with a single "interior" point at an arbitrary amplitude and location - provides the best fit to the data. With a broad, uninformative prior we find the change in the logarithmic evidence ratio is $\Delta \log Z=1$.6. However, using a prior that includes information from our investigation of the Planck data alone, which disfavors knots at $k_{\text {knot }} \gtrsim 10^{-2} \mathrm{Mpc}^{-1}$ or dramatic changes in amplitude, we infer an evidence ratio of $\Delta \log Z=3.1$, a "significant" to "strong" detection according to conventional model selection criteria.

Consequently, this analysis would suggest that BICEP2 has actually made three significant discoveries about inflation: the first is to confirm its existence, and the second is to show that it took place at a relatively high energy scale. Thirdly, these results also suggest that the primordial scalar spectrum has a nontrivial structure which is inconsistent with simple models of inflation. Alternatively, the tensor spectrum may differ from the "standard" inflationary form, due to either a variant model of inflation [23] or a mechanism that is independent of inflation $[12-14,17,20]$. Of course, the conservative explanation of our findings is that they point to tension between the Planck and BICEP2 datasets which will be resolved by more complete analyses and/or additional data. 


\section{Method}

\subsection{Likelihoods, priors, and Bayesian evidence}

We combine the $B$-mode results from BICEP2 $[1,2]$ with temperature and lensing data from Planck [5]. We use the Planck likelihood code [24] with Commander, CamSpec, and lensing likelihood files for the data-likelihood evaluation. We use the COSMO++ library [25] to combine the modified form of the primordial scalar power spectrum (Fig. 2) with the Planck likelihood code and calculate the CMB angular power spectra with CLASS [26, 27]. We employ multimodal nested sampling for parameter estimation and the computation of evidence, using the publicly available code MultiNest [28-30].

We use model posterior probabilities and Bayesian evidences to compare the statistical significance of two competing reconstruction models. This approach penalizes models with parameters for which the likelihood is large only in small regions of parameter space and protects against overfitting the scalar spectrum with too many knots or bins. This approach has previously been employed for power spectrum reconstruction [31-36] and gives conservative and robust assessments of possible physical features in the data.

We use the posterior probability $P(\mathcal{M} \mid$ Data $)$ to assess the statistical significance of a model $\mathcal{M}$. If two models $\mathcal{M}_{i}$ and $\mathcal{M}_{j}$ have the same prior probability, Bayes' theorem yields the relative betting odds between the models via the Bayes factor

$$
B_{i j}=\frac{P\left(\text { Data } \mid \mathcal{M}_{i}\right)}{P\left(\text { Data } \mid \mathcal{M}_{j}\right)},
$$

where the Bayesian evidence (marginalized likelihood) is

$$
P\left(\text { Data } \mid \mathcal{M}_{i}\right)=\int P\left(\theta \mid \mathcal{M}_{i}\right) \mathcal{L}(\text { Data } \mid \theta) d \theta
$$

for the model parameters $\theta$. Here, $\mathcal{L}($ Data $\mid \theta)$ is the data-likelihood and $P\left(\theta \mid \mathcal{M}_{i}\right)$ is the parameter prior probability. Equation (2.2) is a function of our choice of prior, so to be conservative we allow the parameters that define the power spectrum features to vary over a wide range of values in order to thoroughly search the parameter space [37]. Because there is little likelihood for features at $k \gtrsim 10^{-3} \mathrm{Mpc}^{-1}$, a model with more parameters must produce a very large improvement in the likelihood relative to a featureless scenario for evidence to yield "betting odds" that strongly support the more complex model. Consequently, we also examine the improvement in fit $(\Delta \mathcal{L})$ at the maximum likelihood point for each scenario.

We use uniform priors for all of the standard cosmological parameters $\Omega_{b} h^{2}, \Omega_{c} h^{2}, h$, and $\tau$, as well as the 14 "nuisance" parameters in the CAMSPEC likelihood. For convenience, we set the prior probability distribution for $r$ to the posterior distribution obtained by BICEP2 $[1,2]$, which is equivalent to the direct evaluation of the underlying likelihood with a uniform prior on $r$. Consistent with BICEP2, we assume a flat tensor power spectrum and a pivot scale of $k_{*}=0.05 \mathrm{Mpc}^{-1}[1,40]$. We check that we recover the joint constraints on $n_{s}$ and $r$ and the marginalised posterior for the running index reported by BICEP.

\subsection{Non-power-law scalar spectrum}

We perform a complete marginalization of the Planck temperature and lensing likelihood, varying the remaining $\Lambda \mathrm{CDM}$ variables and the full set of Planck "nuisance parameters." We calculate the Bayesian evidence ratios and combine this with the parameter posterior 


\begin{tabular}{c|cc}
\hline \hline $\log ($ Posterior Odds $)$ & Jeffreys Scale & Cosmology Scale \\
\hline 0.0 to 1.0 & Not worth more than a bare mention \\
1.0 to 2.5 & Substantial & Weak \\
2.5 to 5.0 & Strong & Significant \\
$>5$ & Decisive & Strong \\
\hline \hline
\end{tabular}

Table 1. Rough guideline for Bayesian evidence interpretation with the Jeffreys scale [38] and the more conservative "cosmology scale" from Ref. [39]. The posterior odds equal the Bayes factor when the prior models odds are equal.

\begin{tabular}{c|c|c}
\hline \hline \multicolumn{3}{c}{ Broad Priors } \\
\hline Linear Spline & Cutoff & Running \\
\hline$-6<\log _{10} k_{\text {knot }}<0$ & $-6<\log _{10} k_{\text {cut }}<0$ & $-0.1<\alpha_{s}<0.1$ \\
$-2<\log \left(10^{10} \Delta_{\text {knot }}^{2}\right)<4$ & & \\
\hline \hline
\end{tabular}

\begin{tabular}{c|c}
\hline \hline \multicolumn{2}{c}{ Informative Priors } \\
\hline Linear Spline & Cutoff \\
\hline$-6<\log _{10} k_{\text {knot }}<-2$ & $-6<\log _{10} k_{\text {cut }}<-2$ \\
$2<\log \left(10^{10} \Delta_{\text {knot }}^{2}\right)<4$ & \\
\hline \hline
\end{tabular}

Table 2. Priors for the non-standard scalar power spectrum: the location $k_{\text {knot }}$ of the knot, the amplitude of the knot's dimensionless scalar power spectrum $\Delta^{2}$, the running of the spectral index $\alpha_{s}$, and the cutoff scale $k_{\text {cut }}$. The values for $r$ are drawn from the BICEP2 likelihood $\mathcal{L}_{\text {BICEP2 }}$. All cosmological parameters not listed above, as well as the amplitudes at the endpoints, have the same priors as used in our previous analysis, Ref. [22].

probabilities to give a conservative model-selection guideline. We use a scale-invariant primordial tensor spectrum and draw $r$ from a prior defined by the BICEP2 posterior, as noted previously.

We summarize all power spectrum priors in Table 2. We reconstruct the primordial scalar power spectrum in the range $10^{-6} \mathrm{Mpc}^{-1}<k<1.0 \mathrm{Mpc}^{-1}$, using a generalization of the "knot-spline" procedure, developed in Refs [22, 31-35, 41-44]. This process is illustrated in Fig. 2. A complete discussion is given in Ref. [22], but it can be summarised as follows:

1. Fix the endpoints at $k_{\min }=10^{-6} \mathrm{Mpc}^{-1}$ and $k_{\max }=1.0 \mathrm{Mpc}^{-1}$, but allow their amplitudes $A_{\min }$ and $A_{\max }$ to vary, with logarithmic prior, in the ranges $-2<A<4$, where $A=\log \left(10^{10} \Delta_{\zeta}^{2}\right)$

2. Add a "knot" with logarithmic prior in $k$, between $k_{\min }<k_{\text {knot }}<k_{\max }$ and allow its amplitude $A_{\text {knot }}$ to vary in the same ranges as the endpoints in Step 1. 


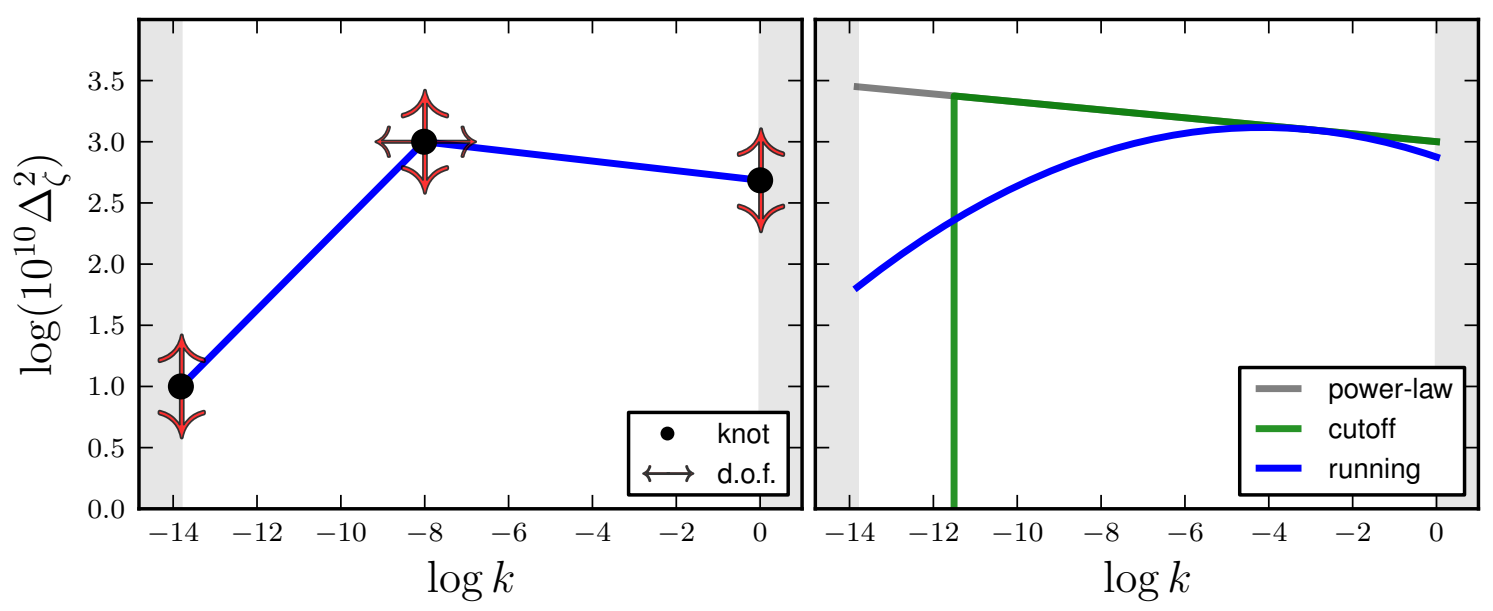

Figure 2. (Left) The knot-spline model for the primordial scalar power spectrum with one knot. The interior knot adds two degrees of freedom, while the endpoints add one each, for a total of four. (Right) The standard power-law, the power-law cutoff at scale $k_{\text {cut }}$, and a power-law with running.

3. Interpolate between the endpoints and the knot with linear-spline interpolation.

Varying the knot-location compensates for the "look-elsewhere" effect, as the knot can move over the whole range of $k$. This permits the reconstruction of features in the scalar primordial power spectrum that could appear at any scale in $k$. The broad ranges on the priors for $k_{\text {knot }}$ and $A_{\text {knot }}$ indicate that possible features are not restricted to large scales. We call this the "broad" prior on the knot's position and amplitude and it gives conservative values for the Bayesian evidence.

We can also use the information gained during our Planck-only analysis [22] to update the prior on the knot position and amplitude. The Planck data indicates that features should only appear at large scales with $k_{\text {knot }} \lesssim 10^{-3}-10^{-2} \mathrm{Mpc}^{-1}$. This reduces the (logarithmic) range of the knot position to two-thirds of the original volume. Furthermore, we know the spectral index is red at large scales, and a low value of $A_{\text {knot }}$ will generate a blue spectrum at $k \gtrsim 10^{-3} \mathrm{Mpc}^{-1}$, so we can further stipulate that $2<A_{\mathrm{knot}}<4$. This gives the Informative Prior for the broken spectrum, which yields less conservative and more significant Bayesian evidences. The Planck likelihood is almost zero in the excluded regions, so the evidence for the informative prior relative to the broad prior is scaled by the ratio of the relative parameter space volume, giving an increase of $\Delta \log Z \approx 1.5$. In what follows we report evidence values with both priors.

We also analyze the standard power-law spectra with both (a) a sharp cutoff at $k_{\text {cut }}$ and (b) a running spectral index, defined by

$$
\Delta^{2}(k)=A_{s}\left(\frac{k}{k_{*}}\right)^{n_{s}-1+\frac{1}{2} \alpha_{s} \log \frac{k}{k_{*}}},
$$

where $\alpha_{s}=d n_{s} / d \log k$ is the running. A running spectral index was considered in the BICEP2 analysis [1]; we repeat this analysis both as a check on our inclusion of the BICEP2 results in our likelihood and in order to calculate Bayesian evidence for the running index.

These two forms of the power spectrum are also illustrated in Fig. 2. The pivot scale for the power law is $k_{*}=0.05 \mathrm{Mpc}^{-1}$. The cutoff prior is from a logarithmic prior, $k_{\text {cut }} \in$ 


\begin{tabular}{c|ccc}
\hline \hline Model & $\Delta \log Z_{\text {Broad }}$ & $\Delta \log Z_{\text {Informative }}$ & $2 \Delta \log \mathcal{L}_{\text {max }}$ \\
\hline No Knots & - & - & - \\
1 Knot & 1.6 & 3.1 & 6.2 \\
\hline \hline Model & $\Delta \log Z_{\text {Broad }}$ & $\Delta \log Z_{\text {Informative }}$ & $2 \Delta \log \mathcal{L}_{\max }$ \\
\hline$\Lambda \mathrm{CDM}+r$ & - & - & - \\
Cutoff & 0.2 & 0.6 & 1.9 \\
Running & 1.1 & - & 3.8 \\
\hline \hline
\end{tabular}

Table 3. Increase in Bayesian evidence, $\Delta \log Z$, and best-fit likelihood, $2 \Delta \log \mathcal{L}_{\text {max }}$, compared to the standard power law case without running. The reported likelihoods $\mathcal{L}_{\max }$ are the product of the Planck and BICEP2 likelihoods.

\begin{tabular}{c|ccccc}
\hline \hline & $\Lambda$ CDM & No knots & 1 knot & Cutoff & Running \\
\hline$\Omega_{b} h^{2}$ & $0.02225_{-0.00030}^{+0.00031}$ & $0.02232_{-0.0033}^{+0.00031}$ & $0.02225_{-0.00030}^{+0.00031}$ & $0.02223_{-0.0030}^{+0.00032}$ & $0.02266_{-0.00035}^{+0.00038}$ \\
$\Omega_{c} h^{2}$ & $0.1174_{-0.0029}^{+0.0029}$ & $0.1167_{-0.0029}^{+0.0030}$ & $0.1175_{-0.0030}^{+0.0030}$ & $0.1173_{-0.0028}^{+0.0028}$ & $0.1162_{-0.0032}^{+0.0032}$ \\
$h$ & $0.690_{-0.014}^{+0.014}$ & $0.693_{-0.014}^{+0.014}$ & $0.689_{-0.014}^{+0.014}$ & $0.690_{-0.013}^{+0.013}$ & $0.698_{-0.016}^{+0.016}$ \\
$\tau$ & $0.092_{-0.028}^{+0.030}$ & $0.100_{-0.028}^{+0.028}$ & $0.101_{-0.030}^{+0.031}$ & $0.094_{-0.028}^{+0.027}$ & $0.118_{-0.033}^{+0.035}$ \\
$r$ & $0.150_{-0.032}^{+0.036}$ & $0.149_{-0.033}^{+0.038}$ & $0.178_{-0.044}^{+0.057}$ & $0.149_{-0.034}^{+0.037}$ & $0.180_{-0.043}^{+0.050}$ \\
$n_{s}$ & $0.9679_{-0.0084}^{+0.0086}$ & - & - & $0.9682_{-0.0082}^{+0.0033}$ & $0.9668_{-0.0096}^{+0.0099}$ \\
$A_{s}$ & $3.086_{-0.051}^{+0.054}$ & - & - & $3.090_{-0.051}^{+0.048}$ & $3.143_{-0.062}^{+0.063}$ \\
$\alpha_{s}$ & - & - & - & - & $-0.024_{-0.010}^{+0.010}$ \\
$\log k_{\text {cut }}$ & - & - & - & $-10_{-2}^{+2}$ & - \\
$A_{\text {min }}$ & - & $3.428_{-0.064}^{+0.069}$ & $1.89_{-1.50}^{+0.74}$ & - & - \\
$A_{\text {max }}$ & - & $3.010_{-0.072}^{+0.071}$ & $3.003_{-0.077}^{+0.078}$ & - & - \\
$\log k_{\text {knot }}$ & - & - & $-5.38_{-5.12}^{+0.69}$ & - & - \\
$A_{\text {knot }}$ & - & - & $3.212_{-0.063}^{+0.107}$ & - & - \\
\hline \hline
\end{tabular}

Table 4. $68 \%$ CI parameter constraints from the reconstruction with different models for the scalar power spectrum. The pivot scale is $k_{*}=0.05 \mathrm{Mpc}^{-1}$. All of the $A$ values are $A=\log \left(10^{10} \Delta^{2}\right)$.

$\left[1.0 \times 10^{-6}, 1.0\right] \mathrm{Mpc}^{-1}$ and we have a uniform prior on $\alpha_{s}$ with the range $\alpha_{s} \in[-0.1,0.1]$. Note that when the running becomes large the Taylor expansion in Eq. (2.3) can be an inaccurate parametrization for the inflationary power spectrum [45]. As above, we also use an Informative Prior for the cutoff spectrum based on the analysis in Ref. [22] with $k_{\text {cut }}<10^{-2} \mathrm{Mpc}^{-1}$. We report the evidences for both priors, although this makes little difference to the conclusions. 

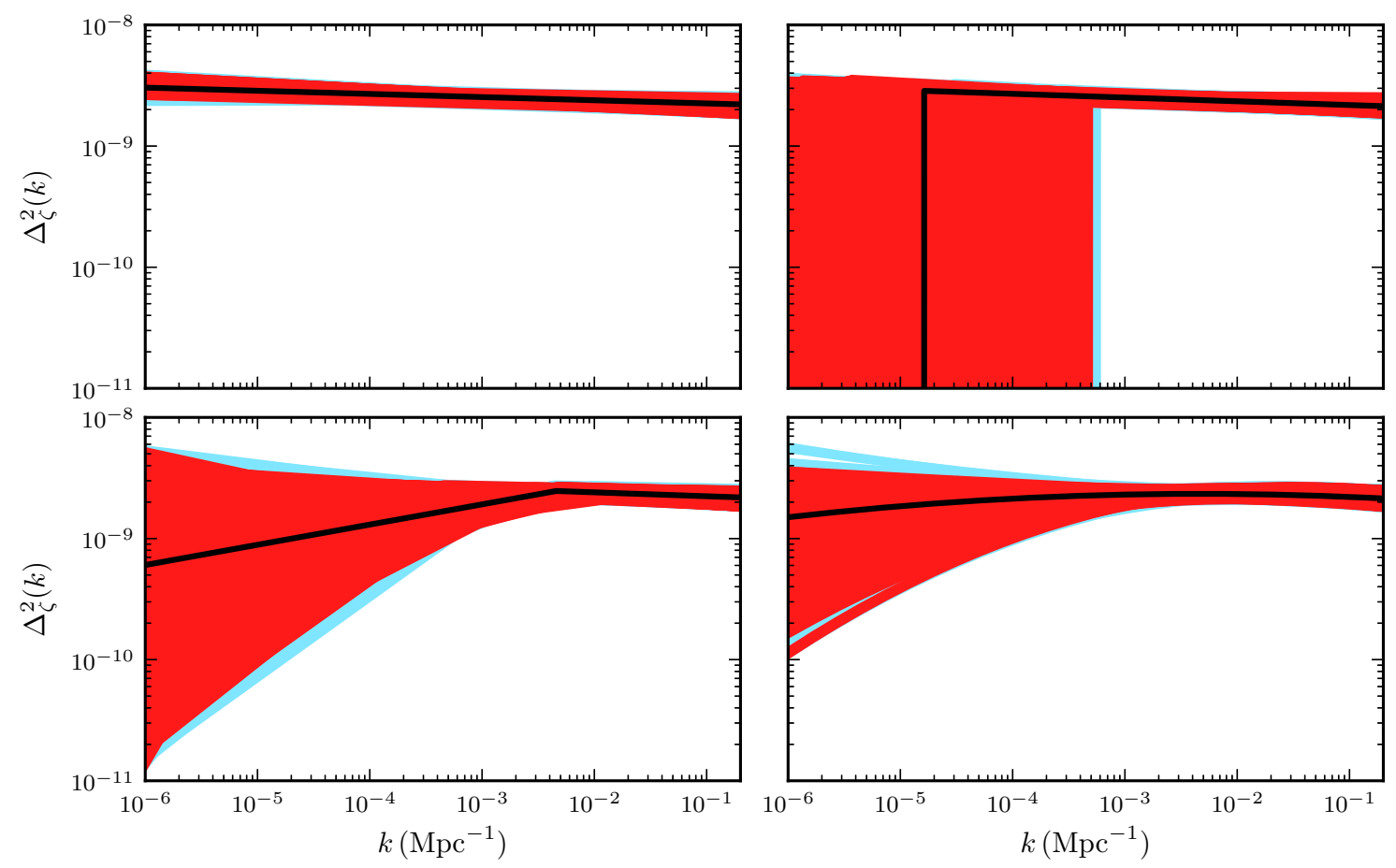

Figure 3. (Left column) The reconstructed primordial power spectrum with (top) 0 and (bottom) 1 knot. (Right column) The power-law spectrum with a (top) cutoff and (bottom) running. The black solid lines show the best-fit, the red lines are the $68 \%$ CI, and the light blue lines are the $95 \%$ CI.

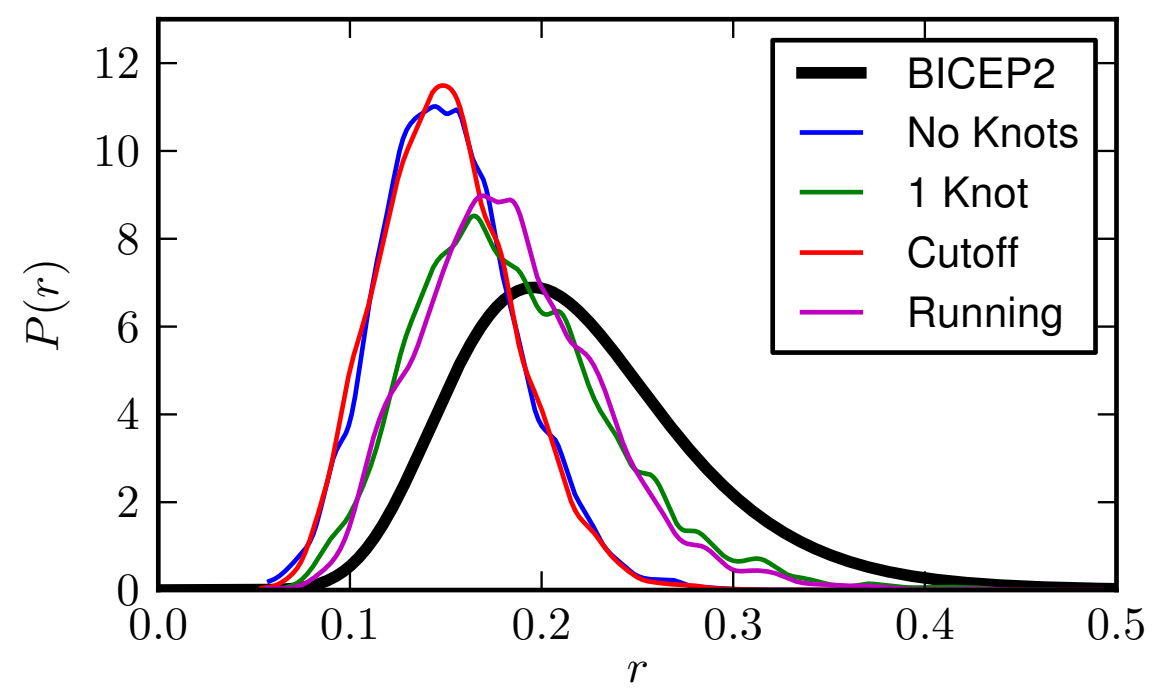

Figure 4. The posterior distributions for the tensor-to-scalar ratio $r$ from the reconstruction with different models for the scalar power spectrum. For comparison, we also show the BICEP2 $[1,2]$ likelihood for $r$. 


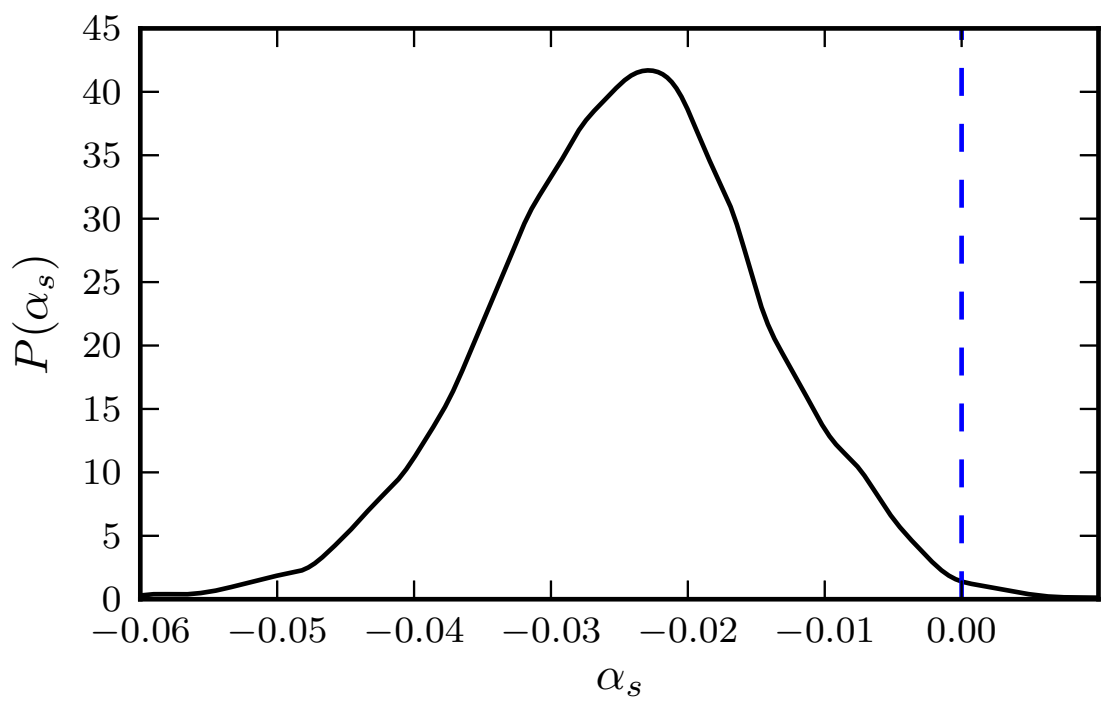

Figure 5. The posterior distribution for the running of the spectral index.

\section{Results}

Figure 3 shows the reconstructed scalar power spectrum with a standard power-law (0 knots); a power-law with a sharp cutoff; a power-law with a running spectral index; and a 1-knot linear-spline model. The Bayesian evidences are given in Table 3, along with the improvements in best fit improvements for each case. Although the linear spline with no knots is equivalent to the standard power law case without running, the different parameterisations lead to different prior volumes. Consequently, we report the Bayesian evidence for the cutoff and running power spectra relative to the standard power law case with common priors for $A_{s}$ and $n_{s}$, while we report the Bayesian evidence for the 1-knot model compared to the 0-knot power-law model.

In Figure 4 we show the posterior distributions for $r$ obtained with these models, along with the result obtained with the BICEP2 data alone. The posterior distribution for $\alpha_{s}$ derived for the running power spectrum is shown in Figure 5. Constraints for the standard cosmological parameters and the power spectrum parameters are displayed in Table $4 .{ }^{2}$ As noted previously while the spline model with no knots is equivalent to $\Lambda \mathrm{CDM}$, the different priors associated with the power spectrum parameterisations lead to small differences in ranges for the standard cosmological parameters. Our 68\% CI bounds for $\alpha_{s}$ agree well with those reported in the BICEP2 analysis $\left(\alpha_{s}=-0.022 \pm 0.010\right)$ [1].

The posteriors for the reconstructed power spectrum in Fig. 3 all recover the standard power-law form at small scales $k \gtrsim 10^{-3} \mathrm{Mpc}^{-1}$. This confirms the Planck-only analysis of Ref. [22], indicating that the BICEP2 detection of $r \sim 0.2$ does not further imply power spectrum features at intermediate to small scales.

At larger scales $\left(k \lesssim 10^{-3} \mathrm{Mpc}^{-1}\right)$ all the non-minimal models indicate a suppression of power in the spectrum of scalar perturbations. The black lines in Fig. 3 are the most likely power spectra, and these all decrease at small $k$. The increase in the likelihood for

\footnotetext{
${ }^{2}$ Although the no knots model is completely equivalent to the standard $\Lambda$ CDM model in its functional form, the parametrizations and subsequently the priors are different. For this reason the resulting posteriors on all of the parameters could be slightly different, and we present the results for both cases.
} 
the best-fit power spectra are reported in Table 3, although we caution that cosmic variance is important at these scales. Also, while a local feature at $k \sim 10^{-3} \mathrm{Mpc}^{-1}$ could also yield posteriors with large-scale power suppression (as shown in Section 4 of Ref. [22]), we can be more certain about the posteriors in Fig. 3: since the tensor contribution to $C_{l}^{T T}$ is nearly uniform for scales $l \lesssim 100$, offsetting this increase in power should require a compensating decrease at all scales and not a local feature at intermediate scales.

The Bayesian evidences in Table 3 show some support for the 1-knot linear-spline model and a running spectrum with $\Delta \log Z=1.6$ and 1.1, respectively. With the Planck temperature and lensing data alone, the evidence for the 1-knot model is only $\Delta \log Z=0.7$ [22], indicating that BICEP2 data contributes significantly to the increased evidence. While these models give qualitatively similar spectra, the running power-law requires that there is less power at scales $k \sim 10^{-3} \mathrm{Mpc}^{-1}$ in order to achieve the same suppression of large-scale power as the 1-knot model. The $C_{l}^{T T}$ at these scales are well-explained by scalar contributions only, but the increased likelihood due to large-scale suppression is partially off-set by the Planck likelihood at $l \sim 15$. This increases the Bayesian evidence for the 1-knot model compared to the running. The cut-off model gives little overall improvement to the data, since a cutoff causes a large decrease in the $C_{l}^{T T}$ over all scales larger than the cut-off scale, as seen in Fig. 1. The 1-knot model generalizes the cut-off at large scales, and thus gives higher evidence values.

Overall, the Bayesian evidences with the broad, uninformative priors show only a mild increase over the power-law $\Lambda \mathrm{CDM}+r$ prediction. The evidence computed for the 1-knot model can be characterized as either "weak" or "substantial," depending whether one uses the Jeffreys or cosmology scale, as described in Table 1. However, the Informative Prior obtained by incorporating the insight gained from our analysis of the Planck data on its own has a significantly higher evidence due to the reduced parameter volume. This increases $\log Z$ by $\sim 1.5$, and the resulting evidence of 3.1 constitutes "strong" or "significant" evidence for the suppression of power in the spectrum of primordial perturbations at large scales.

\section{Discussion}

Using data from Planck and BICEP2 we perform parameter estimates and calculate Bayesian evidence in order to explore the implications of the BICEP2 result for different parameterisations of the primordial scalar power spectrum. In agreement with the BICEP2 analysis we find that the posterior probability for a running spectral index excludes zero at the $95 \%$ confidence interval. Similarly, a spectrum defined by a linear spline with one internal knot shows a distinct preference for a suppression of power in the scalar spectrum at large angular scales, $k \lesssim 10^{-3} \mathrm{Mpc}^{-1}$. For an informative prior incorporating the results of the Planckonly analysis [22] the corresponding Bayesian model selection criteria show a pronounced preference for a model with broken scale invariance, with $\Delta \ln Z=3.6$.

This paper extends the analysis of Ref. [22], which reconstructs the primordial scalar power spectrum from Planck temperature data alone. In particular, Ref. [22] shows that the evidence for extra structure in the scalar power spectrum is negligible and that this reconstruction technique successfully recovers artificial signals injected into simulated temperature maps. Consequently, these results quantify the impact of the additional information provided by BICEP2.

If the scalar power spectrum is not well described by the usual power-law form, the estimated values of other novel cosmological parameters may also be modified, including sum 
of the neutrino masses or the number of effective relativistic degrees of freedom, which can be degenerate with features in the scalar power spectrum [44, 46-50]. Likewise, the BICEP2 data does not put robust constraints on the tensor index $n_{T}$. Generically, the expectation from inflation is that $n_{T}=-r / 8$ to first order in slow-roll, so we assume a scale-invariant tensor spectrum in this analysis. However, while a blue (or sharply peaked) tensor background would also alleviate the tension between Planck and BICEP2, the physical processes that generated this spectrum would at least be as radical as considering a non-power-law scalar spectrum.

The recent BICEP2 result provides strong evidence for a primordial tensor background, from which it is inferred that the very early universe underwent an inflationary phase. However, the results presented here imply that BICEP2 also suggests that this inflationary phase yields a non-trivial scalar power spectrum, and that the underlying inflationary mechanism is not well-described by a simple, smooth single-field potential. The inverse problem associated with reconstructing the inflationary potential from data has been widely discussed [51-69] and these methods would have at least three nontrivial input parameters in such a scenario. Likewise, with a large negative running similar to the central value found here, simple inflationary models typically yield an unacceptably small number of e-foldings, implying that the running itself must be scale dependent [70].

Needless to say, this analysis takes both the current Planck and BICEP2 data-products at face-value. The most conservative explanation for these result is that future analyses will eliminate much of the apparent tension between BICEP2 and other cosmological datasets. From this perspective our analysis quantifies the extent of that tension.

\section{Acknowledgments}

We acknowledge the contribution of the NeSI high-performance computing facilities and the staff at the Centre for eResearch at the University of Auckland, especially Mark Gahegan and Gene Soudlenkov. New Zealand's national facilities are provided by the New Zealand eScience Infrastructure (NeSI) and funded jointly by NeSI's collaborator institutions and through the Ministry of Business, Innovation \& Employment's Research Infrastructure programme [http://www.nesi.org.nz]. KNA is supported by NSF CAREER Grant No. PHY-11-59224.

\section{References}

[1] BICEP2 Collaboration, P. A. R. Ade et. al., BICEP2 I: Detection Of B-mode Polarization at Degree Angular Scales, arXiv:1403.3985 astro-ph.CO (2014).

[2] BICEP2 Collaboration, P. A. R. Ade et. al., BICEP2 II: Experiment and Three-Year Data Set, arXiv:1403.4302 astro-ph.CO (2014).

[3] The POLARBEAR Collaboration, P. Ade et. al., A Measurement of the Cosmic Microwave Background B-Mode Polarization Power Spectrum at Sub-Degree Scales with POLARBEAR, arXiv: 1403.2369.

[4] U. Seljak and M. Zaldarriaga, Signature of gravity waves in polarization of the microwave background, Phys.Rev.Lett. 78 (1997) 2054-2057, [astro-ph/9609169].

[5] Planck Collaboration, P. Ade et. al., Planck 2013 results. I. Overview of products and scientific results, arXiv:1303.5062.

[6] Planck Collaboration, P. Ade et. al., Planck 2013 results. XVI. Cosmological parameters, arXiv: 1303.5076. 
[7] Planck Collaboration, P. Ade et. al., Planck 2013 results. XXII. Constraints on inflation, arXiv: 1303.5082.

[8] SPT Collaboration, Z. Hou et. al., Constraints on Cosmology from the Cosmic Microwave Background Power Spectrum of the 2500-square degree SPT-SZ Survey, arXiv (Dec., 2012) [arXiv: 1212.6267].

[9] ACT Collaboration, S. Das et. al., The Atacama Cosmology Telescope: Temperature and Gravitational Lensing Power Spectrum Measurements from Three Seasons of Data, arXiv (Jan., 2013) [arXiv:1301.1037].

[10] WMAP Collaboration, G. Hinshaw et. al., Nine-Year Wilkinson Microwave Anisotropy Probe (WMAP) Observations: Cosmological Parameter Results, Astrophys.J.Suppl. 208 (2013) 19, [arXiv: 1212.5226].

[11] M. J. Mortonson and U. Seljak, A joint analysis of Planck and BICEP2 B modes including dust polarization uncertainty, arXiv:1405.5857.

[12] U. Seljak, U.-L. Pen, and N. Turok, Polarization of the microwave background in defect models, Phys.Rev.Lett. 79 (1997) 1615-1618, [astro-ph/9704231].

[13] L. Pogosian and M. Wyman, B-modes from cosmic strings, Phys.Rev. D77 (2008) 083509, [arXiv:0711.0747].

[14] K. Jones-Smith, L. M. Krauss, and H. Mathur, A Nearly Scale Invariant Spectrum of Gravitational Radiation from Global Phase Transitions, Phys.Rev.Lett. 100 (2008) 131302, [arXiv: 0712.0778].

[15] T. Kobayashi and F. Takahashi, Running Spectral Index from Inflation with Modulations, JCAP 1101 (2011) 026, [arXiv: 1011.3988].

[16] J. L. Cook and L. Sorbo, Particle production during inflation and gravitational waves detectable by ground-based interferometers, Phys.Rev. D85 (2012) 023534, [arXiv:1109.0022].

[17] L. Senatore, E. Silverstein, and M. Zaldarriaga, New Sources of Gravitational Waves during Inflation, arXiv:1109.0542.

[18] N. Barnaby, J. Moxon, R. Namba, M. Peloso, G. Shiu, et. al., Gravity waves and non-Gaussian features from particle production in a sector gravitationally coupled to the inflaton, Phys.Rev. D86 (2012) 103508, [arXiv: 1206.6117].

[19] D. Carney, W. Fischler, E. D. Kovetz, D. Lorshbough, and S. Paban, Rapid field excursions and the inflationary tensor spectrum, JHEP 1211 (2012) 042, [arXiv:1209.3848].

[20] C. R. Contaldi, M. Peloso, and L. Sorbo, Suppressing the impact of a high tensor-to-scalar ratio on the temperature anisotropies, arXiv:1403.4596.

[21] M. Czerny, T. Kobayashi, and F. Takahashi, Running Spectral Index from Large-field Inflation with Modulations Revisited, arXiv:1403.4589.

[22] G. Aslanyan, L. C. Price, K. N. Abazajian, and R. Easther, The Knotted Sky I: Planck constraints on the primordial power spectrum, arXiv:1403.5849.

[23] V. Miranda, W. Hu, and P. Adshead, Steps to Reconcile Inflationary Tensor and Scalar Spectra, arXiv:1403.5231.

[24] P. Collaboration, Planck 2013 results. XV. CMB power spectra and likelihood, arXiv:1303.5075 (2013).

[25] G. Aslanyan, Cosmo++: An Object-Oriented C++ Library for Cosmology, arXiv:1312.4961 astro-ph.IM (2013).

[26] J. Lesgourgues, The Cosmic Linear Anisotropy Solving System (CLASS) I: Overview, arXiv:1104.2932 (2011). 
[27] D. Blas, J. Lesgourgues, and T. Tram, The Cosmic Linear Anisotropy Solving System (CLASS). Part II: Approximation schemes, Journal of Cosmology and Astroparticle Physics 2011 (2011), no. 07034.

[28] F. Feroz and M. P. Hobson, Multimodal nested sampling: an efficient and robust alternative to Markov Chain Monte Carlo methods for astronomical data analyses, Monthly Notices of the Royal Astronomical Society 384 (2008), no. 2 449-463.

[29] F. Feroz, M. P. Hobson, and M. Bridges, MultiNest: an efficient and robust Bayesian inference tool for cosmology and particle physics, Monthly Notices of the Royal Astronomical Society 398 (2009), no. 4 1601-1614.

[30] F. Feroz, M. P. Hobson, E. Cameron, and A. N. Pettitt, Importance Nested Sampling and the MultiNest Algorithm, arXiv:1306.2144 (2013).

[31] M. Bridges, A. Lasenby, and M. Hobson, A bayesian analysis of the primordial power spectrum, Mon.Not.Roy.Astron.Soc. 369 (2006) 1123-1130, [astro-ph/0511573].

[32] M. Bridges, A. Lasenby, and M. Hobson, WMAP 3-year primordial power spectrum, Mon.Not.Roy.Astron.Soc. (2006) [astro-ph/0607404].

[33] M. Bridges, F. Feroz, M. Hobson, and A. Lasenby, Bayesian optimal reconstruction of the primordial power spectrum, arXiv:0812.3541.

[34] J. A. Vazquez, M. Bridges, M. P. Hobson, and A. N. Lasenby, Model selection applied to reconstruction of the Primordial Power Spectrum, Journal of Cosmology and Astroparticle Physics 06 (2012), no. 0006.

[35] J. A. Vazquez, A. Lasenby, M. Bridges, and M. Hobson, A Bayesian study of the primordial power spectrum from a novel closed universe model, Mon.Not.Roy.Astron.Soc. 422 (2012) 1948-1956, [arXiv:1103.4619].

[36] J. A. Vazquez, M. Bridges, Y.-Z. Ma, and M. Hobson, Constraints on the tensor-to-scalar ratio for non-power-law models, JCAP 1308 (2013) 001, [arXiv:1303.4014].

[37] R. Easther and H. Peiris, Bayesian Analysis of Inflation II: Model Selection and Constraints on Reheating, arXiv (Dec., 2011) [arXiv:1112.0326].

[38] H. Jeffreys, Theory of Probability. Oxford University Press, 1961.

[39] M. P. Hobson, A. H. Jaffe, A. R. Liddle, P. Mukherjee, and D. Parkinson, Bayesian methods in cosmology. Cambridge University Press, 2010.

[40] B. Audren, D. G. Figueroa, and T. Tram, A note of clarification: BICEP2 and Planck are not in tension, arXiv:1405.1390.

[41] L. Verde and H. V. Peiris, On minimally parametric primordial power spectrum reconstruction and the evidence for a red tilt, Journal of Cosmology and Astroparticle Physics 07 (2008), no. 0 009 .

[42] H. V. Peiris and L. Verde, The shape of the primordial power spectrum: A last stand before Planck data, Physical Review D 81 (2010), no. 221302.

[43] S. Bird, H. V. Peiris, M. Viel, and L. Verde, Minimally Parametric Power Spectrum Reconstruction from the Lyman-alpha Forest, Mon.Not.Roy.Astron.Soc. 413 (2011) 1717-1728, [arXiv: 1010.1519].

[44] R. de Putter, E. V. Linder, and A. Mishra, Inflationary Freedom and Cosmological Neutrino Constraints, arXiv:1401.7022 astro-ph.CO (2014).

[45] K. Abazajian, K. Kadota, and E. D. Stewart, Parameterizing the power spectrum: Beyond the truncated Taylor expansion, JCAP 0508 (2005) 008, [astro-ph/0507224].

[46] D. K. Hazra, A. Shafieloo, and T. Souradeep, Cosmological parameter estimation with 
free-form primordial power spectrum, Physical Review D 87 (2013), no. 1123528.

[47] A. Shafieloo and T. Souradeep, Assumptions of the primordial spectrum and cosmological parameter estimation, New Journal of Physics 13 (2011), no. 13024.

[48] S. L. Bridle, A. M. Lewis, J. Weller, and G. Efstathiou, Reconstructing the primordial power spectrum, Monthly Notice of the Royal Astronomical Society 342 (2003), no. 4 L72-L78.

[49] M. Tegmark and M. Zaldarriaga, Separating the early universe from the late universe: Cosmological parameter estimation beyond the black box, Physical Review D 66 (2002), no. 1 103508.

[50] W. H. Kinney, How to fool cosmic microwave background parameter estimation, Physical Review D 63 (2001), no. 443001.

[51] E. J. Copeland, E. W. Kolb, A. R. Liddle, and J. E. Lidsey, Observing the inflaton potential, Phys.Rev.Lett. 71 (1993) 219-222, [hep-ph/9304228].

[52] E. J. Copeland, E. W. Kolb, A. R. Liddle, and J. E. Lidsey, Reconstructing the inflation potential, in principle and in practice, Phys.Rev. D48 (1993) 2529-2547, [hep-ph/9303288].

[53] J. E. Lidsey, A. R. Liddle, E. W. Kolb, E. J. Copeland, T. Barreiro, et. al., Reconstructing the inflation potential : An overview, Rev.Mod.Phys. 69 (1997) 373-410, [astro-ph/9508078].

[54] S. H. Hansen and M. Kunz, Observational constraints on the inflaton potential, Mon.Not.Roy.Astron.Soc. 336 (2002) 1007-1010, [hep-ph/0109252].

[55] S. M. Leach, A. R. Liddle, J. Martin, and D. J. Schwarz, Cosmological parameter estimation and the inflationary cosmology, Phys.Rev. D66 (2002) 023515, [astro-ph/0202094].

[56] S. M. Leach and A. R. Liddle, Microwave background constraints on inflationary parameters, Mon.Not.Roy.Astron.Soc. 341 (2003) 1151, [astro-ph/0207213].

[57] S. M. Leach and A. R. Liddle, Constraining slow - roll inflation with WMAP and 2dF, Phys.Rev. D68 (2003) 123508, [astro-ph/0306305].

[58] W. H. Kinney, E. W. Kolb, A. Melchiorri, and A. Riotto, Inflation model constraints from the Wilkinson Microwave Anisotropy Probe three-year data, Phys.Rev. D74 (2006) 023502, [astro-ph/0605338].

[59] H. Peiris and R. Easther, Recovering the Inflationary Potential and Primordial Power Spectrum With a Slow Roll Prior: Methodology and Application to WMAP 3 Year Data, JCAP 0607 (2006) 002, [astro-ph/0603587].

[60] H. Peiris and R. Easther, Slow Roll Reconstruction: Constraints on Inflation from the 3 Year WMAP Dataset, JCAP 0610 (2006) 017, [astro-ph/0609003].

[61] J. Martin and C. Ringeval, Inflation after WMAP3: Confronting the Slow-Roll and Exact Power Spectra to CMB Data, JCAP 0608 (2006) 009, [astro-ph/0605367].

[62] J. Lesgourgues and W. Valkenburg, New constraints on the observable inflaton potential from WMAP and SDSS, Phys.Rev. D75 (2007) 123519, [astro-ph/0703625].

[63] J. Lesgourgues, A. A. Starobinsky, and W. Valkenburg, What do WMAP and SDSS really tell about inflation?, JCAP 0801 (2008) 010, [arXiv:0710.1630].

[64] H. V. Peiris and R. Easther, Primordial Black Holes, Eternal Inflation, and the Inflationary Parameter Space after WMAP5, JCAP 0807 (2008) 024, [arXiv:0805.2154].

[65] P. Adshead and R. Easther, Constraining Inflation, JCAP 0810 (2008) 047, [arXiv:0802.3898].

[66] J. Hamann, J. Lesgourgues, and W. Valkenburg, How to constrain inflationary parameter space with minimal priors, JCAP 0804 (2008) 016, [arXiv:0802.0505]. 
[67] W. H. Kinney, E. W. Kolb, A. Melchiorri, and A. Riotto, Latest inflation model constraints from cosmic microwave background measurements, Phys.Rev. D78 (2008) 087302, [arXiv:0805.2966].

[68] J. Martin, C. Ringeval, and R. Trotta, Hunting Down the Best Model of Inflation with Bayesian Evidence, Phys.Rev. D83 (2011) 063524, [arXiv:1009.4157].

[69] R. Easther and H. V. Peiris, Bayesian Analysis of Inflation II: Model Selection and Constraints on Reheating, Phys.Rev. D85 (2012) 103533, [arXiv:1112.0326].

[70] R. Easther and H. Peiris, Implications of a Running Spectral Index for Slow Roll Inflation, JCAP 0609 (2006) 010, [astro-ph/0604214]. 